\title{
Quantitative modeling of clinical, cellular, and extracellular matrix variables suggest prognostic indicators in cancer: a model in neuroblastoma
}

\author{
Irene Tadeo', Marta Piqueras², David Montaner ${ }^{3}$, Eva Villamón², Ana P. Berbegall ${ }^{2}$, Adela Cañete ${ }^{4}$, Samuel Navarro ${ }^{2}$ \\ and Rosa Noguera ${ }^{2}$
}

BACKGROUND: Risk classification and treatment stratification for cancer patients is restricted by our incomplete picture of the complex and unknown interactions between the patient's organism and tumor tissues (transformed cells supported by tumor stroma). Moreover, all clinical factors and laboratory studies used to indicate treatment effectiveness and outcomes are by their nature a simplification of the biological system of cancer, and cannot yet incorporate all possible prognostic indicators.

METHODS: A multiparametric analysis on 184 tumor cylinders was performed. To highlight the benefit of integrating digitized medical imaging into this field, we present the results of computational studies carried out on quantitative measurements, taken from stromal and cancer cells and various extracellular matrix fibers interpenetrated by glycosaminoglycans, and eight current approaches to risk stratification systems in patients with primary and nonprimary neuroblastoma.

RESULTS: New tumor tissue indicators from both fields, the cellular and the extracellular elements, emerge as reliable prognostic markers for risk stratification and could be used as molecular targets of specific therapies.

CONCLUSION: The key to dealing with personalized therapy lies in the mathematical modeling. The use of bioinformatics in patient-tumor-microenvironment data management allows a predictive model in neuroblastoma.

T clearly distinguish the heterogeneous spectrum of clinical, histological, and molecular markers of cancer, and thereafter determine the markers essential to diagnosing the degree of malignancy and predicting response to therapy, remains a difficult challenge. These essential markers may be elucidated by considering the patient's organism and transformed tissues as holistically interconnected and dependent on microenvironment interactions via biochemical and biophysical signals (1). Mathematical models enable us to integrate measures made at different levels and generate from relatively simple to highly complex computational descriptors of the disease process of human tumors (2). Digitization of clinical data is necessary to deal with the complex hallmarks of cancer (3). To achieve personalized therapy, wellness and image-defined risk factors must be quantified (4). Hanahan and Weinberg have proposed that eight hallmarks of cancer together constitute an organizing principle that provides a logical framework for understanding the remarkable diversity of neoplastic diseases $(5,6)$. A novel ninth hallmark which includes the aspect of physics has recently been emphasized (7). The identification of these hallmarks by quantifying the structural variations in tumor tissues, at diagnosis as well as during tumor progression and after treatment, using whole histological sections or tissue microarrays with the human eye is a challenging process (8). However, the difficulty can be overcome by forming morphometric data to represent the histological texture and classify the structural changes via sophisticated computational methods (9-11). Cancer cell behavior is altered due to an accumulation of both genetic and epigenetic changes that influence a variety of cellular functions. At the same time, the transformed cells are committed in a complex interaction with the surrounding tumor microenvironment. The tumor microenvironment is comprised of a variety of cell types, lying among a dense network of various extracellular matrix (ECM) fibers merged within the interstitial fluid and gradients of several chemical species, which constantly interplay with malignant cells (12). The stromal cells provide a rich source of growth factors and cytokines, and play a crucial role in modifying the existing connective tissue and depositing new matrix. Tissue scaffolds regulate cell behavior and influence tumor progression $(5-7,12,13)$. Biomathematical analysis of promising biomarker candidates, such as genomic, transcriptomic, proteomic, and epigenomic changes, at the tumor tissue level will play an important role in developing a more powerful mathematical modeling of tumor-microenvironment interactions $(14,15)$. The information obtained can then be linked to tumor progression in patients either refractory to current therapy, or who relapse or who might benefit from novel therapies 
at an earlier stage, in addition to, or instead of, current strategies $(16,17)$. Nevertheless, the mathematical integration of information acquired from clinical and tumor microenvironment interactions into a common description is as difficult to achieve as it is to understand the underlying complexities of the tumor biology itself, and requires translational researchers, experimentalists and biomathematicians $(18,19)$. Due to the diverse behavior exhibited by neuroblastoma tumors, an algorithm of clinical, histopathological and genetic factors to stratify risk assessment and delineate therapeutic decisions has

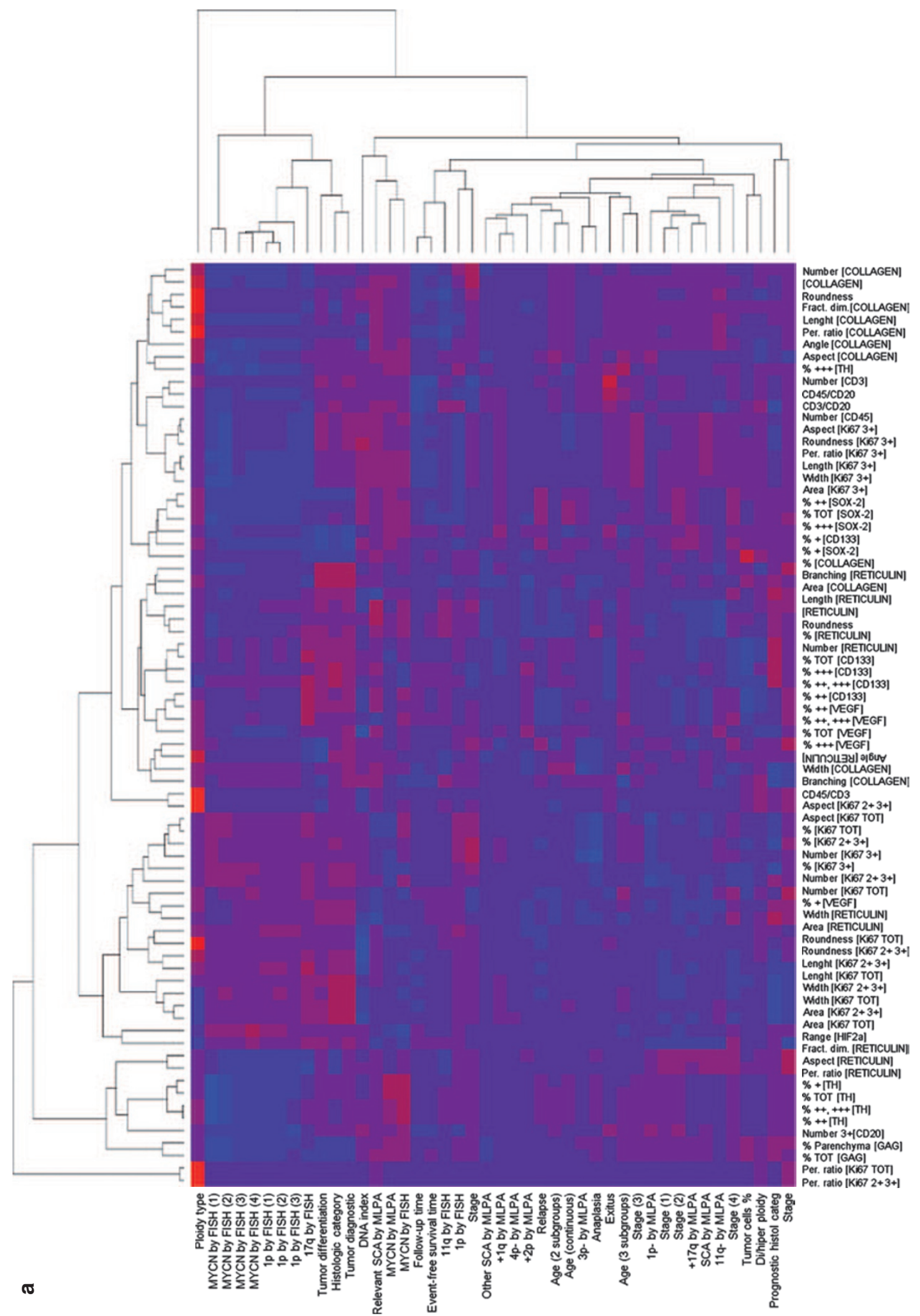




\section{Articles $\mid$ Tadeo et al.}

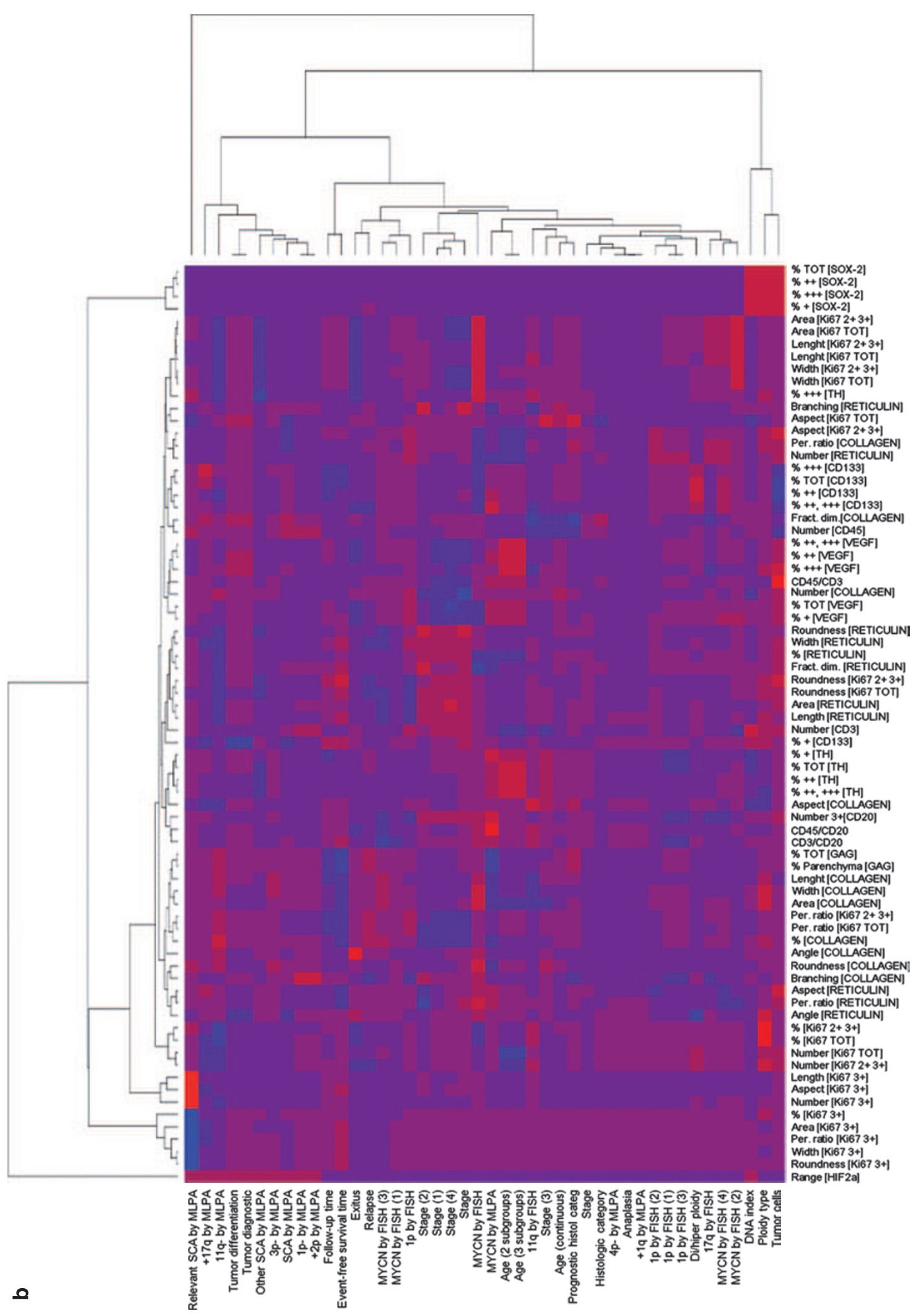

Figure 1. Heatmap clustering indicating the statistical relationship between established prognostic markers and the tumor tissue markers analyzed in (a) primary and (b) nonprimary neuroblastomas. Standard hierarchical clustering showing the correlation in two colors: red (negative) and blue (positive). Statistically significant levels are indicated by the gradient between these two colors; brighter colors represent higher significance. Differences were considered statistically significant for $P$ values $<0.05$. 

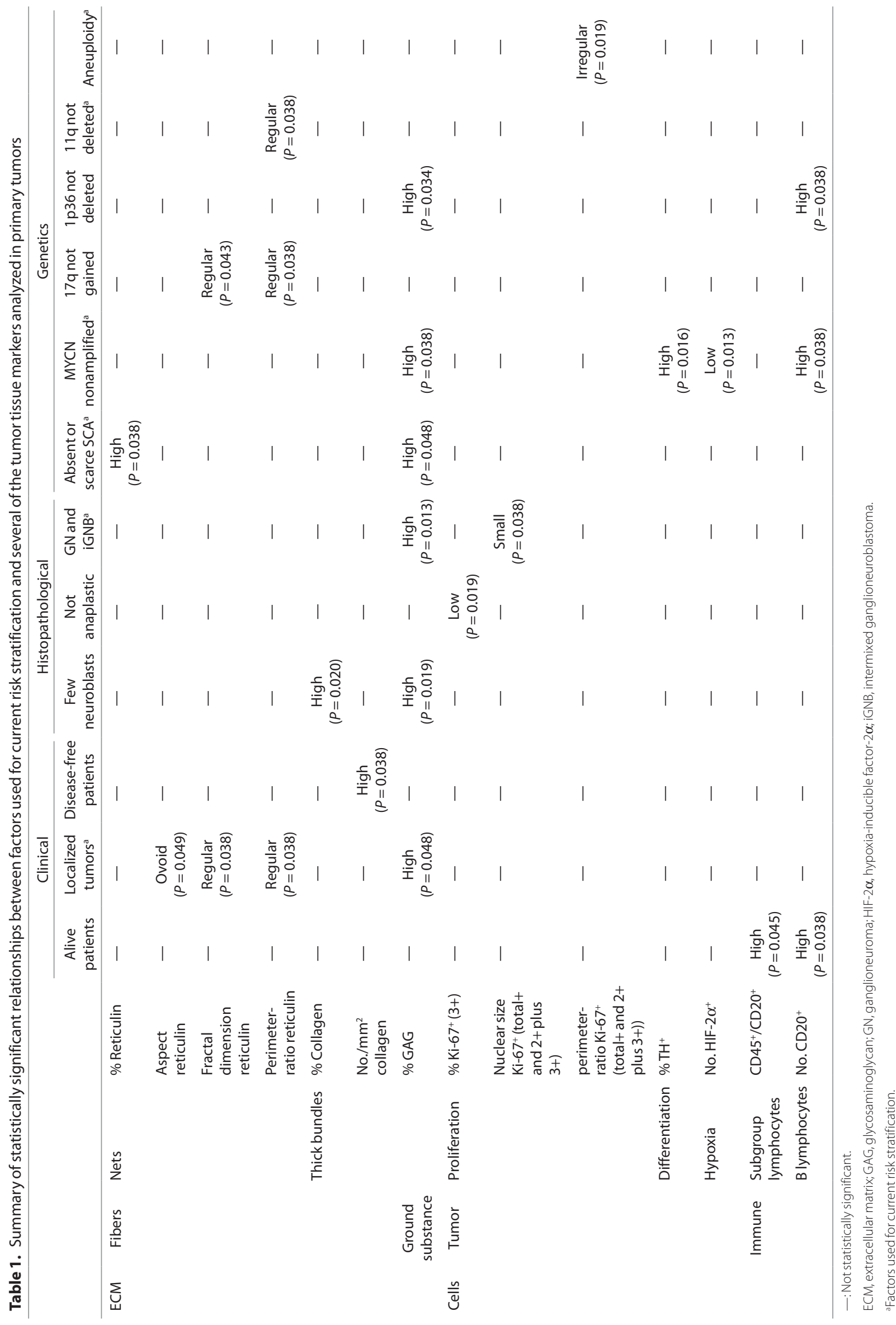


\section{Articles $\mid$ Tadeo et al.}

a
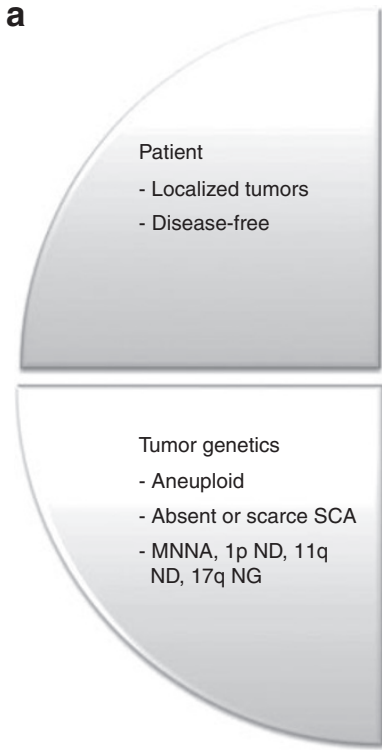

b
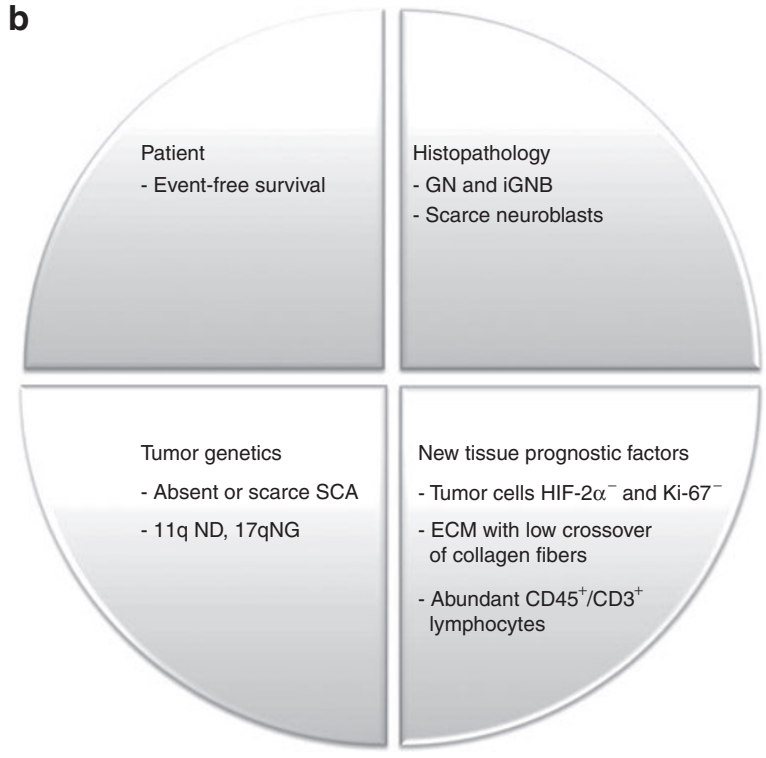

New tissue prognostic factors

- Tumor cells HIF-2 $\alpha^{-}$and $\mathrm{Ki}-67^{-}$ - ECM with low crossover of collagen fibers

- Abundant $\mathrm{CD} 45^{+} / \mathrm{CD}^{+}$ lymphocytes

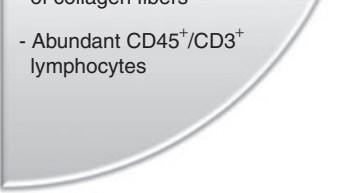

Figure 2. Mathematical modeling of (a) primary and (b) nonprimary neuroblastomas. Synopsis of patient characteristics with better outcome, considering histopathology, tumor genetics, and tumor tissue prognostic factors. ECM, extracellular matrix; GAGs, glycosaminoglycans; GN, ganglioneuroma; iGNB, intermixed ganglioneuroblastoma; SCA, segmental chromosomal aberrations.

been devised. Prognostic systems are based on a combination of patient age at diagnosis (age cut-off $18 \mathrm{mo}$ ); disease stage defined by the International Neuroblastoma Risk Grouping Staging System or the International Neuroblastoma Staging System (20); on histopathological information; MYCN copy number and segmental chromosomal aberrations (SCA) such as $1 \mathrm{p}$ deletion (D) $11 \mathrm{q}$ aberrations or $17 \mathrm{q}$ gain $(\mathrm{G})(21)$; and sometimes DNA ploidy. Accordingly, treatment protocols can vary widely (22). The use of precise quantification by imaging technology of pathology specimens to analyze the structural variations emerging from interactions between cells and ECM elements is a sensitive, objective, and reproducible method that can provide important information for inclusion in computational formulations for risk stratification systems. To illustrate some of these principles, we present the mathematical integration of multimodal data, combining imaging and nonimaging clinical and tumor tissue data, acquired in the context of neuroblastoma studies, suggesting testable hypotheses for making prognostic predictions. Harnessing the synergy of the various forms of data, including clinical variables and biomarker profiles, will allow mathematical predictive models to form part of the holistic approach to medicine in the 21 st century.

\section{RESULTS}

Combined multiparametric analysis gives us an idea about the relationship between the objectively and subjectively quantified histological features and the clinical and genetics data. In Figure 1 the bright colors show some tumor tissue indicators from transformed neuroblastic cells and the cellular and the extracellular compartment, emerging statistically as reliable prognostic markers in primary (PT) and nonprimary tumors (N-PT) (Figure 1a,b respectively).

These results represent an assessment of prognostic criteria based on classical clinical, histological, and growth patterns (age and stage, amount of Schwannian stroma, neuroblastic cell differentiation, and mitosis-karyorrhexis index) and established genetics (MYCN status).

\section{Primary Tumors}

A summary of statistically significant relationships between the established prognostic markers (clinical, histopathological, and genetic parameters) and tumor tissue markers (ECM elements and tumor and immune cells) with good prognostic impact in PT is presented in Table 1. With respect to established prognostic markers and ECM, a high percentage of reticulin fibers were associated with neuroblastomas with absent or scarce SCA, ovoid reticulin nets were related to localized tumors indicating their crosswise arrangement, and regular reticulin nets were present in localized tumors, tumors without $17 \mathrm{q}$ gain $(\mathrm{NG})$ and $11 \mathrm{q}$ nondeleted (ND). In addition, tumors with scant neuroblasts, as well as PT from disease-free patients, were surrounded by a large amount of collagen fibers. A high quantity of glycosaminoglycans was also associated with localized tumors, ganglioneuroma $(\mathrm{GN})$ and intermixed ganglioneuroblastoma or tumors with very few neuroblasts, absent or scarce SCAs, MYCN nonamplified (MNNA) nor 1p36 deletion (D). Biomathematical analyses also revealed that $\mathrm{Ki}-67^{+}$cells from GN and intermixed ganglioneuroblastoma had small nuclei, whereas tumors without anaplasia presented low expression of Ki-67 and aneuploid tumors showed irregular $\mathrm{Ki}-67^{+}$cell nuclei. In addition, MNNA tumors expressed a high amount of $\mathrm{TH}$ and low amount of hypoxia-inducible factor- $2 \alpha$ (HIF- $\left.2 \alpha^{+}\right)$. Regarding immune cells, a higher quantity of $\mathrm{CD} 20^{+}$cells was observed in tumors MNNA, without $1 \mathrm{p} 36$ deletion, and PT from alive patients when compared with MYCN amplification (A), 1p36 D and PT from deceased patients. Figure 2a outlines the characteristics of neuroblastoma patients having a better outcome, considering new and established prognostic markers. 
Table 2. Summary of statistically significant relationships between the established hallmarks for current risk stratification and a few of the tumor tissue markers analyzed in nonprimary tumors

\begin{tabular}{|c|c|c|c|c|c|c|c|c|c|c|}
\hline & & & & \multicolumn{2}{|c|}{ Clinical } & \multicolumn{2}{|c|}{ Histopathological } & \multicolumn{3}{|c|}{ Genetics } \\
\hline & & & & $\begin{array}{l}\text { Alive } \\
\text { patients }\end{array}$ & $\begin{array}{l}\text { Event-free } \\
\text { survival }\end{array}$ & $\begin{array}{c}\text { Few } \\
\text { neuroblasts }\end{array}$ & $\begin{array}{l}\text { GN and } \\
\text { iGNB }^{\mathrm{a}}\end{array}$ & $\begin{array}{l}\text { Absent or } \\
\text { scarce } \\
\text { SCA }^{a}\end{array}$ & $\begin{array}{l}\text { 17q not } \\
\text { gained }\end{array}$ & $\begin{array}{l}\text { 11q not } \\
\text { deleted }^{\mathrm{a}}\end{array}$ \\
\hline ECM & Fibers & Thick bundles & Angle collagen & $\begin{array}{c}\text { Low } \\
(P=0.012)\end{array}$ & - & - & - & - & - & - \\
\hline \multirow[t]{5}{*}{ Cells } & Tumor & Proliferation & $\% \mathrm{Ki}-673^{+}$ & - & - & - & - & $\begin{array}{c}\text { Low } \\
(P=0.000)\end{array}$ & - & $\begin{array}{c}\text { Low } \\
(P=0.027)\end{array}$ \\
\hline & & & Nuclear size Ki-67 3+ & - & $\begin{array}{c}\text { Small } \\
(P=0.003)\end{array}$ & - & - & $\begin{array}{c}\text { Small } \\
(P=0.000)\end{array}$ & $\begin{array}{c}\text { Small } \\
(P=0.019)\end{array}$ & - \\
\hline & & & Aspect Ki-67 3+ & - & $\begin{array}{c}\text { Ovoid } \\
(P=0.004)\end{array}$ & - & - & - & $\begin{array}{c}\text { Ovoid } \\
(P=0.002)\end{array}$ & - \\
\hline & & Hypoxia & No. HIF- $2 \alpha^{+}$ & - & - & - & $\begin{array}{c}\text { Low } \\
(P=0.000)\end{array}$ & - & - & - \\
\hline & Immune & $\begin{array}{l}\text { Subgroup } \\
\text { lymphocytes }\end{array}$ & $\mathrm{CD} 45^{+} / \mathrm{CD}^{+}$ & - & - & $\begin{array}{c}\text { High } \\
(P=0.026)\end{array}$ & - & - & - & - \\
\hline
\end{tabular}

GN, ganglioneuroma; HIF-2 $\alpha$, hypoxia-inducible factor- $2 \alpha$; iGNB, intermixed ganglioneuroblastoma; SCA, segmental chromosomal aberrations.

aFactors used for current risk stratification.

\section{Nonprimary Tumors}

A summary of the statistically significant relationships between the data in N-PT is presented in Table 2. The integration of digitized multimodal data showed that tumors from alive patients presented low crossover of the thick bundles of collagen. In addition, tumors without $11 \mathrm{q} \mathrm{D}$ and with absent or scarce SCA showed a low amount of Ki-67 positive cells. The $\mathrm{Ki}-67^{+}$cells had small, round and irregular nuclei in tumors with $17 \mathrm{q}$ NG, absent or scarce SCA, as well as tumors from patients with long event-free survival. Moreover, GN and intermixed ganglioneuroblastoma had a low amount of HIF$2 \alpha^{+}$cells, whereas tumors with scant neuroblasts presented a high $\mathrm{CD}_{4} 5^{+} / \mathrm{CD}^{+}$cell ratio $(P=0.026)$. Figure $2 \mathrm{~b}$ represents the predictive guide for N-PT in neuroblastoma patients with better outcome.

\section{DISCUSSION}

We outline the benefit to clinical practice of combining established prognostic clinic-biological markers, and medical imaging technology of tumor tissue elements, in a digitized form, using primary and nonprimary neuroblastoma as an example. The hallmarks of cancer constitute an organizing principle for rationalizing the complexities of neoplastic disease. They include sustaining proliferate signaling, evading growth suppressors, avoiding immune destruction, resisting cell death, enabling replicate immortality, inducing angiogenesis, deregulating cellular energetics, activating invasion and metastasis, and physical interactions of cancer cells with their microenvironments (5-7). To study the hallmarks, several concepts have been proposed and considered at different scales of complexity, such as protein interactions, biochemical pathways, cellular functions, or whole organism studies $(2,18)$. The mathematical model in which the whole organism is the principal modeling unit is most intricate and implies the intercorrelation of data from patients, tumor and stromal cells, and extracellular tumor matrix. Clinical data used to construct classifiers in cancer can be transformed into mathematical descriptors, based, at least in part, on medical images for inclusion in whole organism modeling $(1,23,24)$. In fact, in neuroblastoma it has become crucial to evaluate the clinical significance of imaging in detecting PT stage and residual disease in quality-controlled prospective clinical trials. The International Neuroblastoma Risk Grouping has developed a staging system based on preoperative features, since diagnostic images provide more robust and reproducible data than those based on operative findings and approaches (20). This new staging system is not intended to be a substitute for the International Neuroblastoma Staging System, and currently, it is recommended that both systems be used in parallel during a transitional period. We utilized the widely used International Neuroblastoma Staging System to define the tumor stage of our retrospective cohort, based on the age of the patient at diagnosis, local and distant extent of the disease, and the resectability of the tumor. Recognition of the widespread applicability of image-defined risk factors (25) as well as other computational clinical data will increasingly affect the development of mathematical models with 


\section{Articles | Tadeo et al.}

Table 3. Summary of cases with relevant clinical, histopathological, and genetic prognostic factors

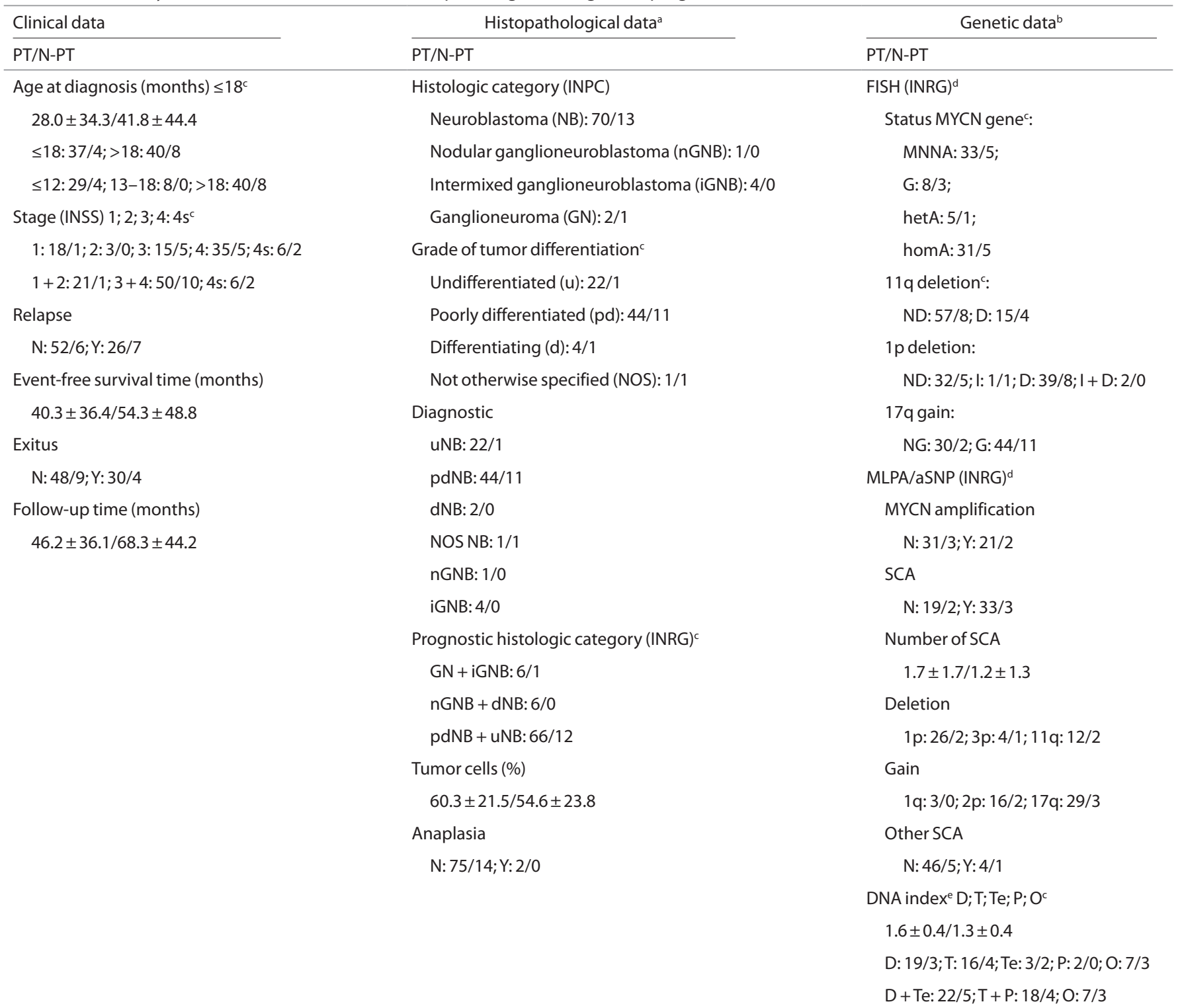

D, diploid; GN, ganglioneuroma; hetA, heterogeneous amplification; homA, homogeneous amplification; I, imbalance; INRG, International Neuroblastoma Risk Group; INPC, International Neuroblastoma Pathology Classification; INSS, International Neuroblastoma Staging System; N, no; N-PT, nonprimary tumors; O, other; P, pentaploid; PT, primary tumors; SCA, segmental chromosomal aberrations; T, triploid; Te, tetraploid; Y, yes.

aThe histology category was determined according INPC (37) and the INRG (22). ${ }^{b}$ Genetic results were interpreted following INRG Biology Committee guidelines (21,34,35); mean \pm

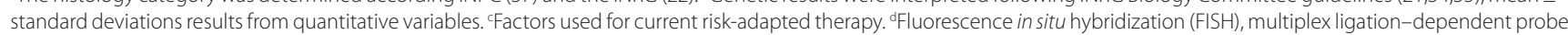
amplification (MLPA), and single-nucleotide polymorphisms arrays (aSNP) were performed to detect genetic aberrations in tumor cells, as previously described (36,38,39). e DNA index was measured using image cytometry.

the whole organism as the modeling unit, and not limited to neuroblastoma alone, but applicable also to other neoplastic diseases.

Technologies for digitizing pathology specimens have advanced significantly in the last decade $(8,11,26)$. Slide scanners are capable of producing high-magnification high-resolution images from whole slides and tissue microarrays within a few minutes. Hence, it is becoming increasingly feasible for basic, clinical and translational research studies to produce thousands of slide images (27). It is fundamental to have a reliable and objective method for precise and quantitative comparison $(28,29)$. We used fast and accurate methods for digitization and objective quantification. Computer-aided evaluation of pathology image analysis to generate risk stratification have been developed for lymphoma (30), glioblastoma (14), breast and prostate cancers (4). A computer-based grading system to support diagnosis for neuroblastoma that uses grades of differentiation and stromal development was published (29,31). Discrimination of stroma-poor and stroma-rich regions has also been reported using a mathematical model performed on digitized hematoxylin-eosin stained wholesections with extraction of textural features (32). Genome 
Table 4. Tissue markers with possible prognostic impact for novel treatment schemes

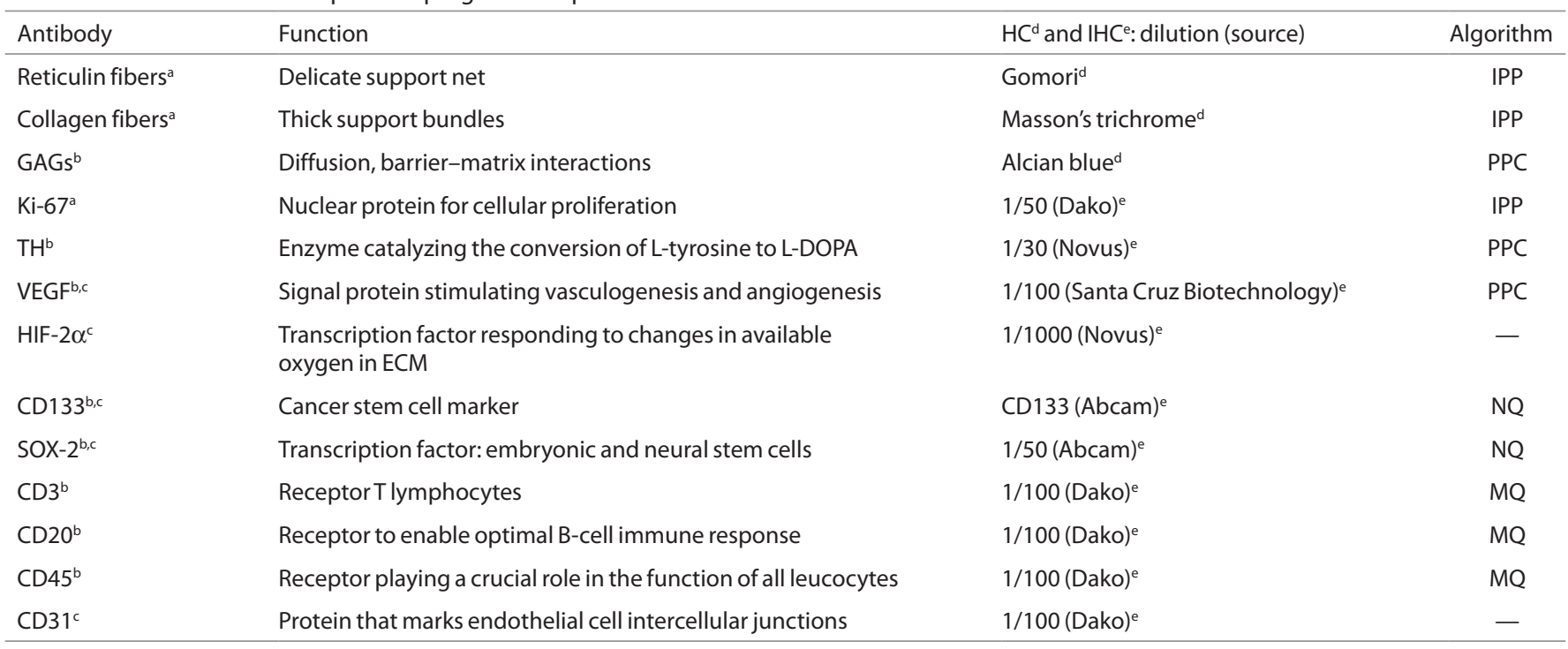

—: Not applied.

CD, cluster of differentiation; CD133, CD133 glycoprotein; CD31, platelet endothelial cell adhesion molecule; GAGs, glycosaminoglycans; HC, histochemistry; HIF-2 $\alpha$, hypoxia-inducible factor-2 $\alpha$; IHC, immunohistochemistry; Ki-67, Ki-67 protein; MQ, Membrane Quantification; NQ, Nuclear Quantification; PPC, Positive Pixel Count; TH, tyrosine hydroxylase; VEGF, vascular endothelial growth factor.

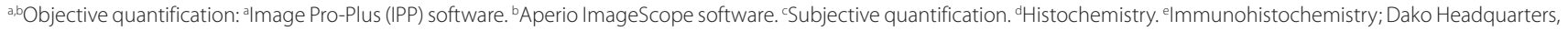
Denmark; Novus Biologicals, Littleton, CO; Santa Cruz Biotechnology, Santa Cruz, CA; Abcam, Cambridge, UK.

instability, inflammation, and physical interactions are recently recognized as characteristics crucial to the acquisition of the cancer hallmarks. It is also clear that much biology is executed by virtue of cells of different types interacting with one another or by interactions with environmental signals from tissue scaffolds or other cells generating phenotypic/genotypic changes $(5-7,12,13)$. The linkage of pathology images with genomics presents an opportunity to study morphology in the context of genetics and patient outcome. For this reason, we consider it important to include not only quantification of grade of tumor differentiation, percentage of tumor cells, protein expression of cancer stem cells and differentiated cancer cells, various immune system cell markers, and amount of vascular structures but also quantification of features of the two major components of the ECM (fibrillar proteins and glycosaminoglycan). The predictive model of these characteristics measured by image analysis techniques with current digitization approaches used for risk stratification system has revealed that the clinical, histopathological, and genetic descriptors that define clinically relevant groups are reflected in the essential characteristics of the cellular morphology and ECM architecture. The mechanical properties of ECM play an important role in governing the behavior of neuroblastic, Schwann, and lymphocyte cells. Our results suggest that Shwannian stroma is not the only important factor in the histopathologic analysis of neuroblastic tumors. Multiparametric analysis of other tumor stroma components (reticulin, collagen fibers, glycosaminoglycan, and immune cells) detected by classic histochemistry (HC) and immunohistochemistry (IHC) techniques and incorporated into a quantitative morphological analysis would improve the International Neuroblastoma Pathology
Classification value. The systematic quantification of a larger digitized cohort of neuroblastoma tumors will resolve difficulties in the following critical areas: consistency of results with less intra- and interobserver variability; assessment of intratumoral histological and genetic heterogeneity; comparative analysis of biomarker expression and genotypic signatures of tumor cells; evaluation of the tumor cell markers obtained by IHC and genetic techniques; and tumor tissue analysis throughout tumor progression. We present a new approach for whole organism modeling of neuroblastoma patients, combining the computational processing of clinicobiological markers used for prognostic stratification and digitized tissue measurements. Advanced high-resolution and high-content imaging technology and computational studies are being developed to integrate knowledge into medical decision support systems (33). We consider that a contribution to the overall goal in cancer would be to construct high-quality bioinformatics strategies to fuse clinical, histologic, and molecular imaging and nonimaging markers for prognosis, response to therapy, and prediction of recurrence in patients, as well as for identifying patient subgroups for inclusion in future clinical trials for new therapeutic compounds. Routine use of digital medical image and computational technologies will allow extraction of parametric information or new hallmarks that could be used as targets of specific therapies in cancer.

\section{METHODS}

\section{Subjects and Prognostic Factors}

Ninety-two tumors from 90 patients (78 original and 14 relapse/ posttreatment) and 4 control tissues included by duplication in two tissue microarrays were used for the study; producing a total of 184 tumor cores and 8 control cores. This study was approved by the 


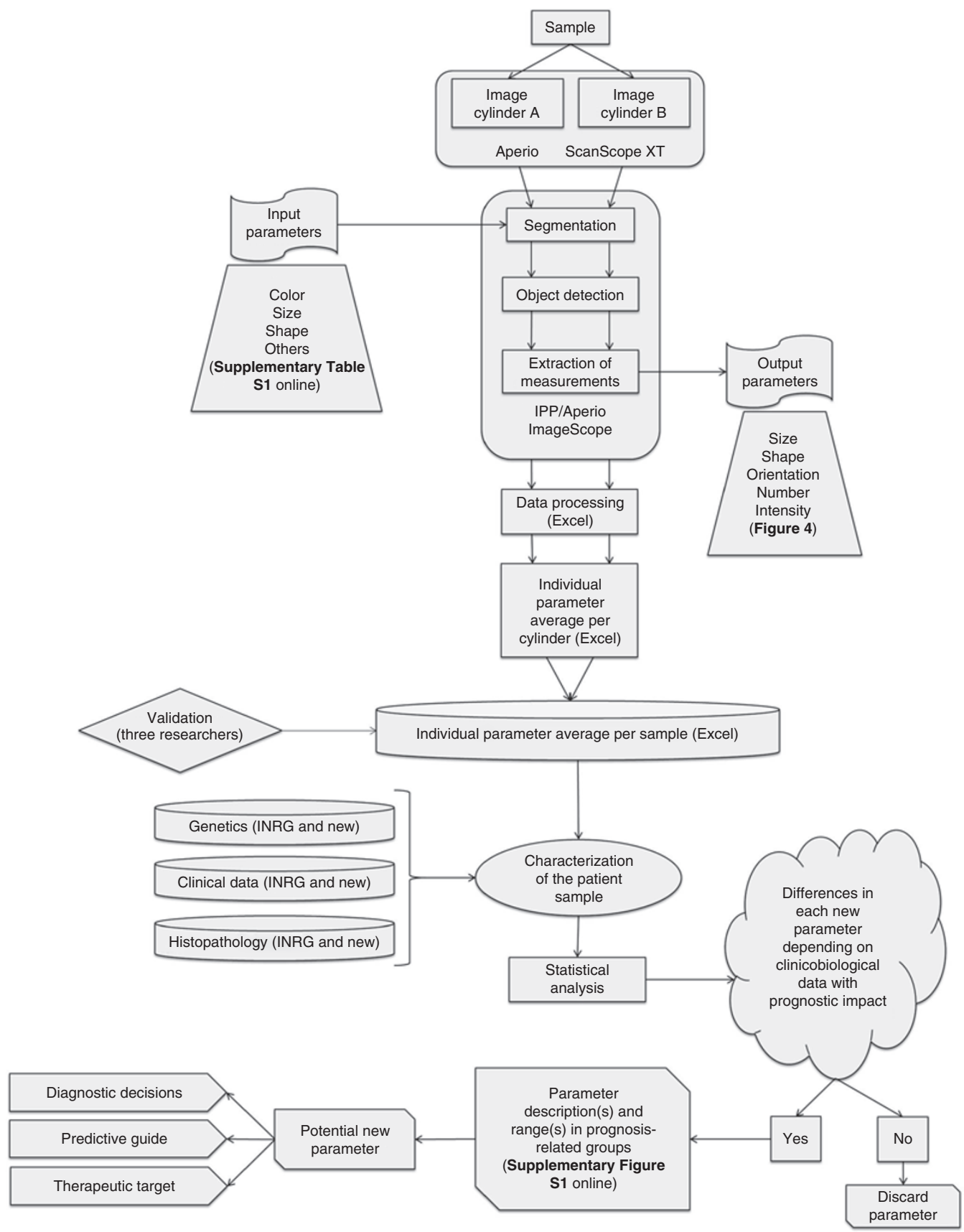

Figure 3. Flowchart showing the multiresolution neuroblastoma image analysis system. Images belonging to different samples stained with different markers have been quantified by image analysis following a common process including segmentation (differential recognition of the staining) with specific input parameters for each marker and method (Supplementary Table S1 online) and extraction of some given parameters (output parameters in Figure 4). The data obtained have been correlated with clinicobiological prognostic data to obtain potential new parameters and ranges for predictive guide, therapeutic decisions, and/or therapeutic target (Supplementary Figure S1 online). IPP, Image Pro-Plus.

Experimental Research Ethics Committee of the Spanish Society of Pediatric Hematology and Oncology (File number: 59C18ABR2002; EC number: 2010-021396-81). Participants or their family members/ informants signed written informed consent forms. Table 3 summarizes the cases with respect to the eight markers currently used for risk-adapted therapy (indicated as ${ }^{c}$ ) $(21,22,34-39)$. In addition, we created new variables related to age, stage, histopathology and genetics. In total, 8 clinical, 6 histopathological, and 17 genetic variables were considered. The hypothetical prognostic factors detected by $\mathrm{HC}$ and IHC, as well as the function of the markers, staining conditions, and quantitative algorithms used, are shown in Table 4.

\section{Image Analysis Measurements}

All the slides were digitized at $40 \times$ magnification using a ScanScope XT scanner (Aperio Technologies, Vista, CA). The image analysis flowchart is showed in Figure 3. Considering the $3 \mathrm{HC}$ and the 10 IHC stains in each tumor core, a total of 2,576 tumor core images were obtained. Two objective methods (1 and 2), and one subjective method (3) were used to quantify the expression of the markers in the digitized images (Figure 4). For objective quantification, different algorithms from image analysis software were applied (Table 4 and Supplementary Table S1 online). Method 1 used self-designed algorithms with the Image Pro-Plus (IPP) software (MediaCybernetics, Warrendale, PA) for noncellular components, such as reticulin and 


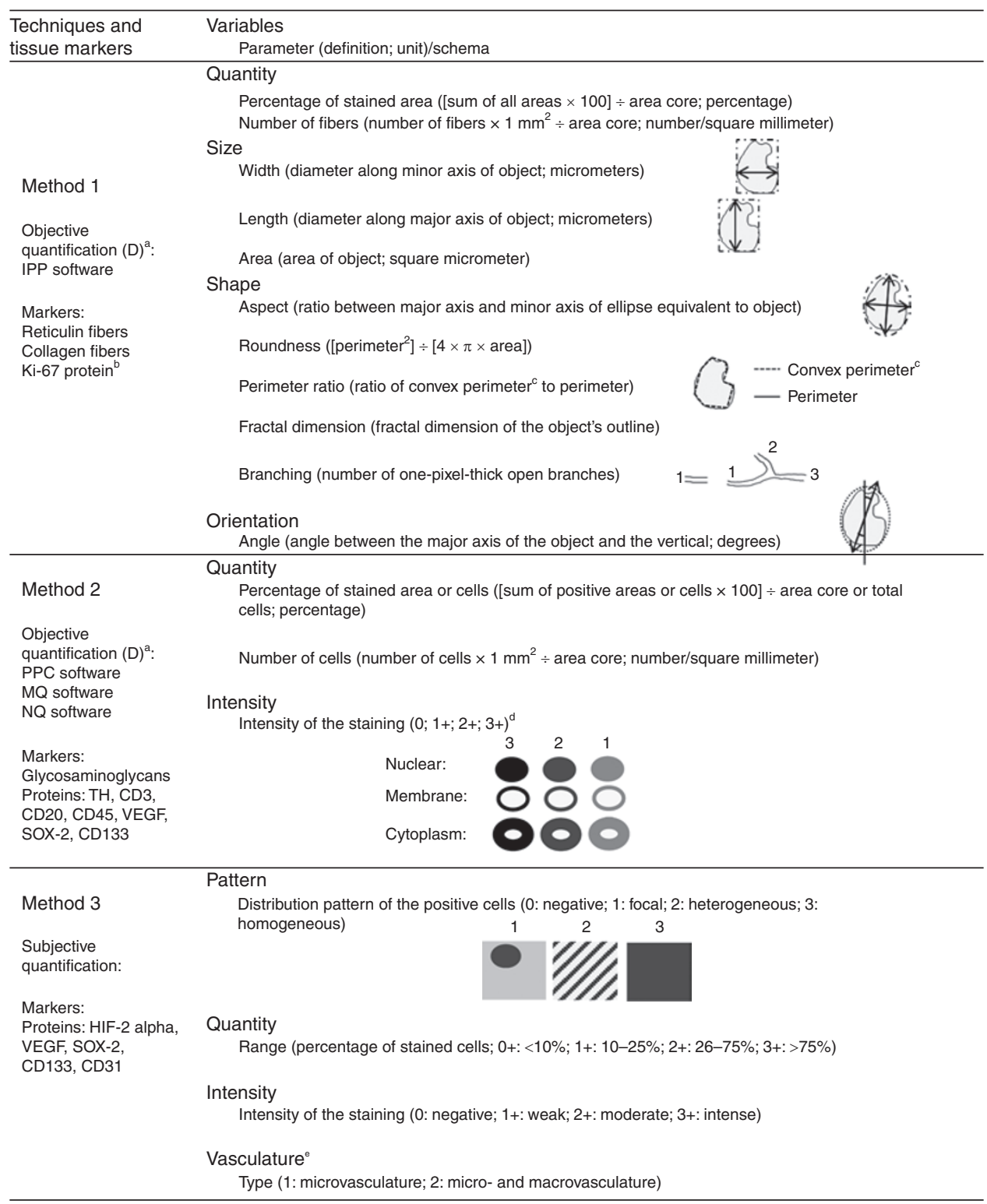

${ }^{\mathrm{a}}$ Digitalization by ScanScope XT scanner at $40 \mathrm{x}$ magnification. ${ }^{\mathrm{b}}$ In all different intensities of staining, the parameters were measured. ${ }^{\mathrm{c}}$ Perimeter of the convex outline of the object. Intensity options of the markers. ${ }^{\mathrm{d}}$ Measured by CD31 marker.

Figure 4. Description of the parameters measured on the tissue markers related to objective (methods 1 and 2) and subjective (method 3) quantification.

collagen fibers, and for cellular proliferation, such as Ki-67 protein expression. This method measured several parameters to describe the quantity (percentage of stained area; number of objects $/ \mathrm{mm}^{2}$ ), the size (width, length, and area), the shape (aspect or how oval the object is; roundness; perimeter ratio or how regular is the surface of the object; fractal dimension or space-filling capacity of a pattern; branching or how many bifurcations has the object), and the orientation (angle) of stained objects. Method 2 applied previously designed algorithms from the Aperio ImageScope software (Aperio Technologies, Vista, CA): a Positive Pixel Count algorithm for total staining area, Membrane Quantification algorithm for membrane staining, and Nuclear Quantification algorithm for nuclear staining. Parameters measured with method 2 estimated the quantity (percentage of stained areas/cells; number of stained cells), and the staining intensity. Finally, the subjective method (method 3) established the pattern (distribution of positive cells), the range (percentage of stained cells), and the staining intensity, as previously described (40), as well as the presence of vascular structures. A summary of objective and subjective measurements, including mean and standard deviation, distinguishing PT and N-PT tumors is shown in Tables 5 and 6 respectively. In total, 23 HC parameters (with objective quantification) plus 28 IHC parameters ( 15 with objective and 13 subjective quantification) were determined in each tumor core analyzed. Four possible options for staining intensity (indicated in Figure 3d) of the IHC markers were considered for each of the 15 objectively quantified parameters. In total, 97 parametric measures from each core were obtained. Given that the 92 analyzed tumors had duplicated cores in tissue microarrays, 17,848 tumor parametric measures were obtained overall.

\section{Statistical Analysis}

For a more complete statistical analysis, in addition to the $23 \mathrm{HC}$ and 28 IHC parameters already defined, seven new variables were created. For IHC immune cell markers, the three ratios $\mathrm{CD} 45^{+} / \mathrm{CD}^{+}$, $\mathrm{CD} 45^{+} / \mathrm{CD} 20^{+}$, and $\mathrm{CD} 20^{+} / \mathrm{CD}^{+}$were also calculated. With regard to subjectively quantified IHC staining parameters, the sum of staining intensity, range, and pattern was created for each analyzed marker, except CD31. Moreover, in the objectively quantified IHC staining 
Articles | Tadeo et al.

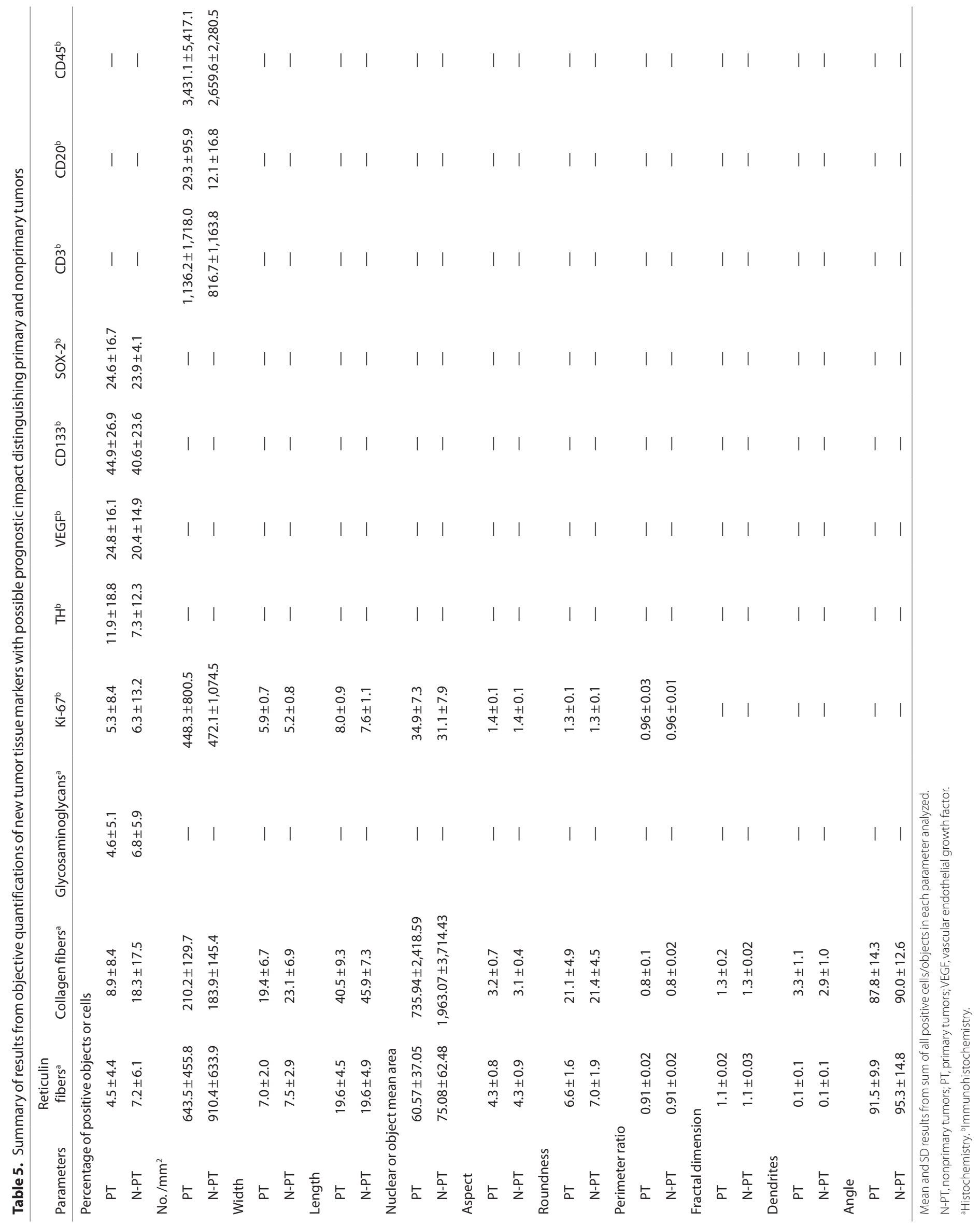


Table 6. Summary of results from subjective quantifications of new tumor tissue markers with possible prognostic impact distinguishing primary and nonprimary tumors

\begin{tabular}{|c|c|c|c|c|c|}
\hline \multirow[b]{2}{*}{ Parameters } & \multicolumn{5}{|c|}{ Number of cases } \\
\hline & VEGF & HIF- $2 \alpha$ & CD133 & SOX-2 & CD31 \\
\hline \multicolumn{6}{|c|}{ Range (0/1/2/3) } \\
\hline N-PT & $5 / 4 / 1 / 2$ & $9 / 1 / 0 / 0$ & $4 / 1 / 1 / 8$ & $5 / 3 / 3 / 3$ & - \\
\hline \multicolumn{6}{|c|}{ Pattern (n/het/hom/f) } \\
\hline N-PT & $5 / 0 / 6 / 1$ & $9 / 1 / 0 / 0$ & $4 / 1 / 1 / 8$ & $5 / 2 / 4 / 3$ & - \\
\hline \multicolumn{6}{|c|}{ Intensity (n/w/m/h) } \\
\hline PT & $13 / 28 / 12 / 18$ & $32 / 13 / 2 / 13$ & $18 / 3 / 8 / 44$ & $28 / 6 / 17 / 22$ & - \\
\hline N-PT & $5 / 4 / 1 / 2$ & $9 / 0 / 0 / 1$ & $4 / 1 / 1 / 8$ & $5 / 2 / 3 / 4$ & - \\
\hline \multicolumn{6}{|c|}{ Type of vasculature (mi/b) } \\
\hline
\end{tabular}

(0) $<10 \%$, (1) 10-25\%, (2) 26-75\%, (3) >75\%; (n) negative, (f) focal, (w) weak, (m) moderate, (h) high; (mi) micro, (b) both macro/micro.

HIF-2 $\alpha$, hypoxia-inducible factor-2 $\alpha ; \mathrm{N}-\mathrm{PT}$, nonprimary tumors; PT, primary tumors; VEGF, vascular endothelial growth factor.

parameters, in addition to the four intensity categories distinguished, two new categories were created: the sum of all positive cells $(1+, 2+$, and $3+$ ), and the sum of $2+$ and $3+$ positive cells. Subsequently, these 58 tissue marker variables (Tables 4-6) plus the 31 variables derived from the patient's clinical history, the histopathology and the tumor genetic information (Table 3 ) were analyzed. Linear regression models were used to assess the dependence of the clinical variables upon genetic and histopathologic markers. All continuous variables were log transformed where necessary to achieve a more normal or symmetric distribution. A regression model was constructed for each analyzed pair of variables. The Wald statistic was used to derive $P$ values accounting for the significance of the slope in the model, being different from zero. Thus, for continuous variables, linear regression models provide the same information as the Pearson correlation. Linear models also have the advantage of being applicable when the independent variable, the marker in our case, is binary or discrete. Thus, we can explore the relationship of our clinical variables with each of our markers, independently of whether the marker is a continuous or a discrete variable. This paradigm is appropriate to our study as half our variables, the genetic ones, are discrete while those derived from imaging are generally continuous. Separate analyses were carried out for PT and N-PT. All $P$ values where corrected for multiple testing to control the false discovery rate. The standard procedure described in Benjamini-Hochberg was used for this study (41). All analyses where done using R statistical software (42). A sample classification would be possible after processing a whole set of data for a single patient (Supplementary Figure S1 online)

\section{SUPPLEMENTARY MATERIAL}

Supplementary material is linked to the online version of the paper at http:// www.nature.com/pr

\section{ACKNOWLEDGMENTS}

We thank Marcial García-Rojo (Pathology Unit, General Hospital, Ciudad Real, Spain) and Javier Chaves (Genetic Diagnosis and Genotyping Unit, Medical Research Foundation INCLIVA, Hospital Clínico Universitario, Valencia, Spain) for excellent technical assistance, SEHOP (Spanish Society of Hematology and Pediatric Oncology) and Désirée Ramal (Paediatric Oncology Unit, University Hospital La Fe, Valencia, Spain) for patient data management, and David Harrison for English language assistance.

\section{STATEMENT OF FINANCIAL SUPPORT}

This study was supported by grants from the Fundación Asociación Española contra el Cáncer; FIS (contract PI10/15) and RTICC (Red Temática de Investig- ación Cooperativa en Cáncer, contracts RD06/0020/0102; RD12/0036/0020), Instituto Carlos III Madrid \& ERDF (European Regional Development Fund), Spain.

Disclosure: The authors declare no conflict of interest.

\section{REFERENCES}

1. Hood L, Balling R, Auffray C. Revolutionizing medicine in the $21^{\text {st }}$ century through systems approaches. Biotechnol J 2012;7:992-1001.

2. Sanga S, Frieboes HB, Zheng X, Gatenby R, Bearer EL, Cristini V. Predictive oncology: a review of multidisciplinary, multiscale in silico modeling linking phenotype, morphology and growth. Neuroimage 2007;37:Suppl 1:S120-34.

3. Smarr L. Quantifying your body: a how-to guide from a systems biology perspective. Biotechnol J 2012;7:980-91.

4. Madabhushi A, Agner S, Basavanhally A, Doyle S, Lee G. Computeraided prognosis: predicting patient and disease outcome via quantitative fusion of multi-scale, multi-modal data. Comput Med Imaging Graph 2011;35:506-14.

5. Hanahan D, Weinberg RA. The hallmarks of cancer. Cell 2000;100:57-70.

6. Hanahan D, Weinberg RA. Hallmarks of cancer: the next generation. Cell 2011;144:646-74.

7. Mierke CT. Physical break-down of the classical view on cancer cell invasion and metastasis. Eur J Cell Biol 2013;92:89-104.

8. García Rojo M. State of the art and trends for digital pathology. Stud Health Technol Inform 2012;179:15-28.

9. Braun M, Kirsten R, Rupp NJ, et al. Quantification of protein expression in cells and cellular subcompartments on immunohistochemical sections using a computer supported image analysis system. Histol Histopathol 2013;28:605-10.

10. Gabril MY, Yousef GM. Informatics for practicing anatomical pathologists: marking a new era in pathology practice. Mod Pathol 2010;23:34958.

11. Laurinavicius A, Raslavicus P. Consequences of "going digital" for pathology professionals - entering the cloud. Stud Health Technol Inform 2012;179:62-7.

12. Noguera R, Nieto OA, Tadeo I, Fariñas F, Alvaro T. Extracellular matrix, biotensegrity and tumor microenvironment. An update and overview. Histol Histopathol 2012;27:693-705.

13. Brábek J, Mierke CT, Rösel D, Veselý P, Fabry B. The role of the tissue microenvironment in the regulation of cancer cell motility and invasion. Cell Commun Signal 2010;8:22. 
14. Cooper LA, Kong J, Gutman DA, et al. Integrated morphologic analysis for the identification and characterization of disease subtypes. J Am Med Inform Assoc 2012;19:317-23.

15. Martínez-González A, Calvo GF, Pérez Romasanta LA, Pérez-García VM. Hypoxic cell waves around necrotic cores in glioblastoma: a biomathematical model and its therapeutic implications. Bull Math Biol 2012;74:2875-96.

16. Cohen JE. Mathematics is biology's next microscope, only better; biology is mathematics' next physics, only better. PLoS Biol 2004;2:e439.

17. Moghaddasi FL, Bezak E, Marcu L. In silico modelling of tumour margin diffusion and infiltration: review of current status. Comput Math Methods Med 2012;2012:672895.

18. Rejniak KA, McCawley LJ. Current trends in mathematical modeling of tumor-microenvironment interactions: a survey of tools and applications. Exp Biol Med (Maywood) 2010;235:411-23.

19. Trisilowati T, Mallet DG. In silico experimental modeling of cancer treatment. ISRN Oncol 2012;2012:828701.

20. Monclair T, Brodeur GM, Ambros PF, et al.; INRG Task Force. The International Neuroblastoma Risk Group (INRG) staging system: an INRG Task Force report. J Clin Oncol 2009;27:298-303.

21. Schleiermacher G, Mosseri V, London WB, et al. Segmental chromosomal alterations have prognostic impact in neuroblastoma: a report from the INRG project. Br J Cancer 2012;107:1418-22.

22. Cohn SL, Pearson AD, London WB, et al.; INRG Task Force. The International Neuroblastoma Risk Group (INRG) classification system: an INRG Task Force report. J Clin Oncol 2009;27:289-97.

23. Frieboes HB, Zheng X, Sun CH, Tromberg B, Gatenby R, Cristini V. An integrated computational/experimental model of tumor invasion. Cancer Res 2006;66:1597-604.

24. Wolkenhauer O, Auffray C, Jaster R, Steinhoff G, Dammann O. The road from systems biology to systems medicine. Pediatr Res 2013;73(4 Pt 2):502-7.

25. Brisse HJ, McCarville MB, Granata C, et al.; International Neuroblastoma Risk Group Project. Guidelines for imaging and staging of neuroblastic tumors: consensus report from the International Neuroblastoma Risk Group Project. Radiology 2011;261:243-57.

26. Prasad K, Prabhu GK. Image analysis tools for evaluation of microscopic views of immunohistochemically stained specimen in medical research-a review. J Med Syst 2012;36:2621-31.

27. Garcia Rojo M, Punys V, Slodkowska J, Schrader T, Daniel C, Blobel B. Digital pathology in Europe: coordinating patient care and research efforts. Stud Health Technol Inform 2009;150:997-1001.

28. Al-Janabi S, Huisman A, Van Diest PJ. Digital pathology: current status and future perspectives. Histopathology 2012;61:1-9.
29. Gurcan MN, Boucheron LE, Can A, Madabhushi A, Rajpoot NM, Yener B. Histopathological image analysis: a review. IEEE Rev Biomed Eng 2009;2:147-71.

30. Sertel O, Lozanski G, Shana'ah A, Gurcan MN. Computer-aided detection of centroblasts for follicular lymphoma grading using adaptive likelihoodbased cell segmentation. IEEE Trans Biomed Eng 2010;57:2613-6.

31. Kong J, Sertel O, Boyer KL, Saltz JH, Gurcan MN, Shimada H. Computerassisted grading of neuroblastic differentiation. Arch Pathol Lab Med 2008;132:903-4; author reply 904.

32. Sertel O, Kong J, Shimada H, Catalyurek UV, Saltz JH, Gurcan MN. Computer-aided Prognosis of Neuroblastoma on Whole-slide Images: Classification of Stromal Development. Pattern Recognit 2009;42:1093103.

33. Bernsen MR, Ruggiero A, van Straten M, et al. Computed tomography and magnetic resonance imaging. Recent Results Cancer Res 2013;187:3-63.

34. Ambros IM, Benard J, Boavida M, et al. Quality assessment of genetic markers used for therapy stratification. J Clin Oncol 2003;21:2077-84.

35. Ambros PF, Ambros IM, Brodeur GM, et al. International consensus for neuroblastoma molecular diagnostics: report from the International Neuroblastoma Risk Group (INRG) Biology Committee. Br J Cancer 2009;100:1471-82.

36. Piqueras M, Navarro S, Castel V, Cañete A, Llombart-Bosch A, Noguera R. Analysis of biological prognostic factors using tissue microarrays in neuroblastic tumors. Pediatr Blood Cancer 2009;52:209-14.

37. Shimada $\mathrm{H}$, Umehara $\mathrm{S}$, Monobe $\mathrm{Y}$, et al. International neuroblastoma pathology classification for prognostic evaluation of patients with peripheral neuroblastic tumors: a report from the Children's Cancer Group. Cancer 2001;92:2451-61.

38. Villamón E, Berbegall AP, Piqueras M, et al. Genetic instability and intratumoral heterogeneity in neuroblastoma with MYCN amplification plus $11 \mathrm{q}$ deletion. PLoS ONE 2013;8:e53740.

39. Villamón E, Piqueras M, Berbegall AP, et al. Comparative study of MLPAFISH to determine DNA copy number alterations in neuroblastic tumors. Histol Histopathol 2011;26:343-50.

40. Gómez-Mateo Mdel C, Piqueras M, Påhlman S, Noguera R, Navarro S. Prognostic value of SOX2 expression in neuroblastoma. Genes Chromosomes Cancer 2011;50:374-7.

41. Benjamini Y, Hochberg Y. Controlling the false discovery rate: A practical and powerful approach to multiple testing. J R Stat Soc Ser B Stat Methodol 1995;57:289-300.

42. R Core Team. R: A language and environment for statistical computing. $\mathrm{R}$ Foundation for Statistical Computing, 2013. (http://r-project.org/.) 